C-reactive protein (CRP) and muscle-associated enzymes creatine phosphokinase (CK) and lactate dehydrogenase (LD) were measured by routine laboratory methods.

Results In patients with PM, serum levels of S100A4 protein were significantly higher than those observed in healthy controls or DM patients $(148.6 \pm 351.5$ versus $80.75 \pm 285.1, p<0.01$ and $43.55 \pm$ $53.03, \mathrm{p}<0.05$, respectively). No significant differences in S100A4 levels were found between CAM patients $(119.9 \pm 414.0)$ and healthy controls or other myositis patients. In the whole group of IIM patients, serum S100A4 levels correlated with MYOACT score $(\mathrm{r}=0.39 ; \mathrm{p}<0.001)$ and its components Constitutional Disease Activity (DA) $(r=0.34 ; \mathrm{p}<0.001)$ and Pulmonary DA $(r=0.44$; $p<0.001)$. Serum S100A4 correlated also with Muscular DA $(r=0.25 ; p<0.05)$, CK $(r=0.33 ; p<0.01)$ and LD $(r=0.40$; $p<0.001)$. S100A4 levels correlated with Cutaneous DA ( $r=0.46$; $\mathrm{p}<0.01$ ) in DM patients and with Extramuscular Global Assessment only in PM patients $(r=0.55 ; \mathrm{p}<0.001)$. No significant correlations of S100A4 serum levels in patients with CAM were found. In the 11 longitudinal IIM samples there was no significant decrease of S100A4 serum levels observed. Multiple regression of the whole IIM patients group showed significant association of S100A4 serum levels with Pulmonary DA $(\beta=0.369 ; p<0.01)$, LD $(\beta=0.345$; $p<0.01)$ and severity of dysphagia $(\beta=-0.250 ; p<0.05)$. In $P M$ patients, S100A4 levels were associated with Extramuscular Global Assessment $(\beta=0.552 ; \mathrm{p}<0.01)$ and in DM patients with MYOACT $(\beta=0.557 ; p<0.01)$ and $\operatorname{CRP}(\beta=0.391 ; p<0.05)$.

Conclusions This is the first study showing that circulating levels of S100A4 are associated with several features of IIM disease activity, particularly with extramuscular components. We did not find any association of S100A4 levels and cancer associated myositis. Further studies analysing bioactive form of S100A4 and the role of S100A4 in cancer associated myositis are needed.

\section{A4.5 DO HIGH MOLECULAR WEIGHT ADIPONECTIN LEVELS ASSOCIATE WITH RADIOGRAPHIC PROGRESSION IN EARLY RHEUMATOID ARTHRITIS AND HAND OSTEOARTHRITIS?}

doi:10.1136/annrheumdis-2013-203217.5

IIR Klein-Wieringa, 'SN Andersen, 'JC Kwekkeboom, 'L Herb-v.Toorn, 'AHM van der Helm-van Mil, 2 I Meulenbelt, 'TWJ Huizinga, 'M Kloppenburg, 'REM Toes, ${ }^{1}$ A loan-Facsinay. 'Dept. of Rheumatology, Leiden University Medical Center, Leiden, The Netherlands; ${ }^{2}$ Dept. of Molecular Epidemiology, Leiden, The Netherlands

Background Adipose tissue can secrete many different soluble factors (adipokines) influencing whole body metabolism. Some of these adipokines, such as adiponectin, have been shown to influence radiographic progression in osteoarthritis $(O A)$ and rheumatoid arthritis (RA). In RA patients, total adiponectin (totAPN) levels in serum associate positively with radiographic progression, which suggestes an adverse effect on disease. Intruiguingly, in patients with hand OA, high totAPN levels in serum associated with a decreased relative risk for radiographic progression, suggesting a benificial effect on disease.

Adiponectin is a pleiotropic adipokine, which consists of several isoforms. Of these isoforms, high molecular weight adiponectin (hmwAPN) has been described as one of the most biologically active and its effect on radiographic progression in $\mathrm{RA}$ and hand $\mathrm{OA}$ is unknown.

Objective Therefore, we explored the possibility that the association between totAPN and disease progression is primarily mediated by the hmwAPN isoform.

Methods Concentrations of hmwAPN and totAPN were determined in baseline plasma of 324 RA patients from the Early Arthritis Cohort (EAC) and in baseline sera of 164 hand OA patients from the Genetics Arthrosis and Progression (GARP) study. The association between levels of hmwAPN and totAPN with radiographic progression were determined using a multivariate normal regression model (EAC cohort) or by generalised estimated equations (GARP cohort). Adjustments were made for age, gender, treatment strategy and Body Mass Index (BMI).

Results In RA patients totAPN associated positively with radiographic progression (Sharp van der Heijde scores) (association estimate $3.65, p=0.002$ ), whereas in patients with hand OA, totAPN associated negatively with radiographic progression (joint space narrowing (JSN)) (Odds 0.24/Odds 0.21, $p=0.002 / p=0.002$ two highest tertiles compared to the lowest tertile). HmwAPN on the other hand, did not associate significantly with radiographic progression in patients with hand OA or RA, although in patients with RA we did observe a trend towards a positive association (association estimate $1.53 p=0.07$ ) upon correcting for age, gender and treatment strategy. This trend was lost after further adjustment for BMI. Similar results were obtained when joint space narrowing (JSN) was used as outcome measurement.

Conclusions Our data further substantiate the connexion between APN-levels and radiographic progression in rheumatic disease and indicate that the differential effects associated between totAPN and radiographic progression in either in $\mathrm{RA}$ and hand $\mathrm{OA}$ is not mediated by (a selective effect of) hmwAPN.

\section{A4.6 EFFECTS OF CHOLIC ACID AND ITS DERIVATIVES IN EXPERIMENTAL ARTHRITIS}

doi:10.1136/annrheumdis-2013-203217.6

'Silvia Hayer, ${ }^{2}$ Emina Halilbasic, 'Birgit Niederreiter, 'Martin Willburger, 'Peter Mandl, 'Victoria Saferding, 'Stephan Blüml, 'Josef Smolen, ${ }^{2}$ Michael Trauner, 'Kurt Redlich. 'Medical University of Vienna, Department of Internal Medicine III, Division of Rheumatology; ${ }^{2}$ Medical University of Vienna, Department of Internal Medicine III, Division of Gasteroenterology

Introduction Bile acids play an important role in cholesterol metabolism and act as intestinal detergents for digestion and absorption of fats and fat-soluble vitamins. Disruption of bile flow causes cholestatic liver diseases. Derivatives of cholic acid (CA) such as nor-ursodeoxycholic acid (norUDCA) are promising therapeutic agents in the treatment of cholangiopathies. Previous studies also demonstrated anti-inflammatory and anti-fibrotic properties of norUDCA in experimental sclerosing cholestasis.

Objective To investigate the anti-inflammatory potential of CA and its derivatives ursodeoxycholic acid (UDCA) and nor-UDCA in in Collagen-induced arthritis (CIA), an animal model for inflammatory, erosive arthritis.

Methods Mice were prophylactically treated with CA, UDCA or nor-UDCA enriched diet pellets $(5 \mathrm{mg} / \mathrm{kg}$ diet $)$ or standard diet pellets (Placebo) ad libitum starting 1 week before the first immunisation with collagen. Animals were weekly assessed for clinical signs of arthritis, body weight and food consumption during the experimental period. After 10 weeks of treatment hind paws, liver, sera and lymph nodes were isolated for further analysis. Sera were investigated for anti-collagen antibodies, cytokine responses and liver parameters such as alkaline phosphatase (AP) and alanine transaminase (ALT). Paraffin-sections of hind paws were examined for histoptahological changes in synovial inflammation, subchondral bone erosion, cartilage damage and osteophyte formation. Cell populations within synovial pannus were identified by immunhistochemical stainings and were determined using HistoQuest software (from TissueGnostics).

Results Uptake of CA, UDCA and norUDCA was confirmed by serum analysis. Prophylactic treatment of CIA mice with UDCA and norUDCA could not significantly prevent disease incidence. In contrast, treatment with CA led to a marked increase in disease incidence and severity compared to Placebo treated animals. Whereas UDCA and norUDCA showed a similar course of clinical signs of 
arthritis. Histological analysis of hind paws revealed an increase in synovial inflammation, bone erosion and cartilage damage in the CA treated animals compared to the Placebo group. No changes in joint pathology were observed in the UDCA or norUDCA treated group. Despite differences in absolute numbers of infiltrating cells, immunhistochemical staining for cell specific markers demonstrated no markedly changes in the distribution of neutrophils, macrophages, T- and B-lymphocytes within inflammatory synovial tissue. Similar amounts of total anti-collagen IgG and its subtypes IgG2 and $\operatorname{IgG} 2 \mathrm{c}$ were found in all treatment groups. Moreover, FACS analysis of lymph node cells revealed similar amounts of T-cells, $\mathrm{B}$-cells, dendritic cells and macrophages. In contrast, serum analyses revealed a trend toward increased IL- 6 levels suggesting that CA exacerbates IL-6 levels driving disease onset and severity in CIA model.

Conclusions Bile acid derivatives UDCA and norUDCA could not modulate collagen-induced arthritis. In contrast, cholic acid (CA) exacerbated the incidence and severity of inflammatory, erosive arthritis in this model. Thus, promising anti-inflammatory and anti-fibrotic agents for cholangiopathies have therefore no beneficial effects in an experimental arthritis model.

\section{A4.7 EVALUATION OF TISSUE INFLAMMATION AND ADIPOKINE EXPRESSION IN GASTRIC AND LUNG TISSUE OF SYSTEMIC SCLEROSIS (SSC) PATIENTS}

doi:10.1136/annrheumdis-2013-203217.7

\begin{abstract}
${ }^{1} \mathrm{~N}$ Lepper, ${ }^{1} \mathrm{M}$ Peters,,${ }^{1,2} \mathrm{M}$ Vasile, ${ }^{3} \mathrm{E}$ Roeb, ${ }^{4} \mathrm{~A}$ Günther, ${ }^{1} \mathrm{U}$ Müller-Ladner, 'E Neumann. 'Dept Internal Medicine and Rheumatology, Justus-Liebig-University Gießen, Kerckhoff Klinik, Bad Nauheim, Germany; ${ }^{2}$ Dept of Clinic and Medical Therapy, Div Rheumatology, Sapienza, University of Rome, Italy; Internal Medicine/Gastroenterology, University of Gießen, Gießen, Germany; ${ }^{4}$ Internal Medicine/Pneumology, University of Gießen, Gießen, Germany
\end{abstract}

Background Adipokines are bioactive substances secreted by adipose tissue and also by different resident cells. Adipokines are known to have influence on metabolism but there is inceasing evidence of the immunomodulatory role of different adipokines including adiponectin, visfatin/PBEF and resistin. For example, these adipokines are strongly expressed in synovial tissue of RA patients compared to controls. Since little is known about the role of adipokines in other rheumatic diseases including SSc, we analysed the expression of the adipokines adiponectin, resistin and visfatin in SSc organ involvement, specifically in gastric and lung tissue of SSc and control patients.

Methods Gastric samples from SSc $(n=9)$ and gastritis $(n=12)$ patients were examined for the expression of adiponectin, visfatin and resistin and also for the immune cell markers CD4, CD8 and CD68 by histology and immunohistochemistry. First, sections from each tissue were examined by hematoxilin/eosin (HE). Thereafter, lung samples from SSc $(n=2)$, IPF (idiopathic pulmonary fibrosis, $\mathrm{n}=8)$, and healthy controls $(\mathrm{n}=8)$ were examined by immunhistochemistry for the expression of adiponectin, visfatin, resistin and surfactant-protein C (SPC). Immune cell and adipokine expression was analysed qualitatively in gastric samples and semiqualitatively in lung samples.

Results The numbers of $\mathrm{CD}^{+}$and $\mathrm{CD}^{+}{ }^{+} \mathrm{T}$-cells in the gastric wall were comparable in SSc $(22.1 \pm 7.3$ resp. $15.3 \pm 2.2)$ and gastritis $(23.8 \pm 3.3$ resp. $15.3 \pm 4.0)$ patients. SSc patients showed a higher number of $\mathrm{CD} 68^{+}$immune cells compared to gastritis patients $(13.3 \pm 2.2$ versus $6.8 \pm 1.9)$, reaching statistical significance $(p=0.036)$. Visfatin and resistin expression in the gastric wall was present in most patients from both groups without significant difference. Gastric adiponectin expression was decreased significantly in SSc compared to gastritis patients $(p=0.049)$ being only present in SSc microvasculature if at all. Adiponectin expression was positively correlated to the number of $\mathrm{CD} 4^{+} \mathrm{T}$-cells and inversely correlated to the number of $\mathrm{CD} 68^{+}$immune cells. Lung samples of healthy and fibrotic (SSc and IPF) tissue showed a intermediate or strong visfatin and resistin expression. The pattern of resistin and visfatin expression was similar in all tissues, being cell-associated and present among bronchial epithel and immune cells, especially within lymphoid aggregates. Adiponectin was expressed in vessels and lung parenchyma, but not by immune cells. Again, adiponectin was decreased significantly within fibrotic lung tissue from SSc and IPF patients compared to healthy controls ( $p<0.0001)$.

Conclusions T-cell involvement in gastritis appears to be linked to inflammation including SSc even prior to onset of fibrosis, potentially enhanced by local macrophages and supported by resistin and visfatin. In contrast, the strong decrease of adiponectin in SSc gastric tissue and SSc and IPF lung tissue supports the idea of a role in fibrosis. Taken together, adipokines appear to be involved in distinct mechanisms of SSc and IPF pathophysiology.

The study was funded by the LOEWE initiative UGMLC: Universities of Giessen \& Marburg Lung Center.

\section{A4.8 GDF15, A MARKER OF LUNG INVOLVEMENT IN SYSTEMIC SCLEROSIS, IS INVOLVED IN ALTERED CYTOKINE SECRETION BY FIBROBLASTS, BUT DOES NOT IMPAIR FIBROSIS DEVELOPMENT}

doi:10.1136/annrheumdis-2013-203217.8

Stijn Lambrecht, Vanessa Smith, Filip De Keyser, Dirk Elewaut. Department of Rheumatology, Ghent University

Objective Systemic sclerosis is a progressive connective tissue involving autoimmune processes. It is generally known that members of the TGF- $\beta$ superfamily are involved in the regulation of connective tissue metabolism in systemic sclerosis (SSc), but also in regulating the immune system. Growth differentiation factor 15 is a distant member of this TGF- $\beta$ family. We aim to evaluate the role of GDF15 in SSc-pathogenesis.

Methods A longitudinal prospective cohort of SSc patients was screened for GDF15 serum levels by ELISA and associations with disease activity and tissue damage were analysed. Moreover, in vitro stimulation experiments were performed in lung fibroblasts. The role of GDF15 in fibrosis development in vivo was evaluated by performing the bleomycin lung fibrosis model in GDF15 deficient mice.

Results Serum samples from a cohort of 122 patients were screened for GDF15 levels. An increase in GDF15 levels was observed in patients classified as limited SSc, limited cutaneous SSc and diffuse SSc. Moreover, GDF15 serum levels highly correlated with disease activity and clinical symptoms of lung fibrosis. This was also mimicked in the bleomycin mouse model of SSc. Here, bleomycin exposed animals displayed elevated expression levels of GDF15 in lung tissue. Isolated lung fibroblast of GDF15 deficient mice showed reduced induction of IL6 and CCL2 upon bleomycin stimulation compared to wild-type littermates. Both, IL6 and CCL2, are involved in recruitment and activation of the immune system. Surprisingly, no differences in end-stage fibrosis development were observed between wild-type and GDF15 deficient animals after bleomycin injection.

Conclusions An intriguing profile of GDF15 serum levels was found in SSc patients. GDF15 expression is induced during fibrosis development. We hypothesise that the altered GDF15 expression by lung fibroblast may contribute to distorted interaction between the immune system and the stromal connective tissue. From our data it is clear that this protein may participate in fibrosis initiation, but is not indispensable in the course of fibrosis development in vivo. 